\title{
Synthesis of Poly(AAm-co-AA) and Investigation of its Swelling Behavior: Using Response Surface Methodology (RSM)
}

\author{
Samira Heidari ${ }^{1}$, Jafar Saberi Doust ${ }^{2}$, Feridun Esmaeilzadeh ${ }^{1 *}$ \\ ${ }^{1}$ School of Chemical and Petroleum Engineering, Enhanced Oil and Gas Recovery Institute, Enhanced Gas Condensate Recovery \\ Research Group, Shiraz University, Shiraz, Iran \\ ${ }^{2}$ Petro Pars Ghodrat Company, Shiraz, Iran \\ Email: *esmaeil@shirazu.ac.ir
}

How to cite this paper: Heidari, S., Doust, J.S. and Esmaeilzadeh, F. (2018) Synthesis of Poly(AAm-co-AA) and Investigation of its Swelling Behavior: Using Response Surface Methodology (RSM). Modeling and $\mathrm{Nu}$ merical Simulation of Material Science, 8, 65-78.

https://doi.org/10.4236/mnsms.2018.84004

Received: September 14, 2018

Accepted: October 28, 2018

Published: October 31, 2018

Copyright $\odot 2018$ by author(s) and Scientific Research Publishing Inc.

This work is licensed under the Creative Commons Attribution-NonCommercial International License (CC BY-NC 4.0).

http://creativecommons.org/licenses/by-nc/4.0/ cc) (i) (8) Open Access

\begin{abstract}
Unwanted water production together with oil and gas production is a striking problem in oil and gas industries, and many approaches have been examined to overcome this major problem. Preformed particle gels (PPGs) showed dramatically good properties for this purpose in mature oil and gas reservoirs. In this study, we carefully synthesized an efficient series of PPGs with using a design of experiments (DOE) software. Acrylamide (AAm)/Acrylic acid (AA) mole ratio, $\mathrm{N}, \mathrm{N}^{\prime}$-methylenebisacrylamide (MBA) mole percentage and swelling time were considered as key parameters to examine PPG swelling behavior. Our results presented a detailed empirical correlation, which could significantly predict the swelling capacity of PPGs in $\mathrm{CaCl}_{2}$ salt solution $(200,000$ ppm).
\end{abstract}

\section{Keywords}

Response Surface Methodology (RSM), Optimization, Synthesis, Water Conformance

\section{Introduction}

Today, the remained oil in the most of oil reservoirs is a heavy oil. Because of increasing demand for energy sources, enhanced oil recovery (EOR) from oil reservoirs has been vastly considered in the worldwide. There are several approaches to upgrade the heavy oil from reservoirs such as gas injection, water flooding, thermal, and chemical methods. In many EOR approaches, it is tried to reduce oil viscosity and literally squeeze oil through the pores of the oil matrix.

Producing unwanted water together with oil is a big trouble for the most of 
producers in many EOR processes. Because unwanted water production increases the corrosion level, environmental worries, burden on fluid-handling facilities and eventually workover costs, it is contrary to the economically oil production. When the amount of unwanted water production exceeds the economic limitations of water oil ratio (WOR), the production is winded up and the well is closed. Hence, a major objective of many oil producers is to control unwanted water production.

There are different ways to control unwanted water production from oil and gas reservoirs; solutions that are relatively inexpensive to more complex. Gel treatment is one of the successful methods to control unwanted water production from fractured oil reservoirs [1] [2] [3]. The gel reduces water production by using two mechanisms: changing wettability [4] [5] or/and blocking fractures [6]. Hitherto, gels have been employed in both immature (gelant) [7] [8] [9] and preformed particle gel (PPG) forms [10] [11]. One of the differences between these two forms is that the crosslinking process of gelant occurs at the reservoir conditions while it occurs in the surface facilities for PPGs. The fundamental discrepancy between the gelant and PPG is that PPG is resistant against all kinds of salt solutions with high concentration, temperature and pressure. This difference makes PPG approach to be superior. In addition, PPG is adjustable in size between micrometers and centimeters, is inflatable between 50 - 200 times of original size, and is controllable in the swelling behavior. Therefore, PPGs are more successful than immature gels [12] [13].

Based on the currently available evidences, the most important parameters that affect the PPG performance are its synthesis approach [14] [15] [16], the properties of salt solution and the properties of medium in where PPG is swollen. Solution and suspension polymerization are two well-known synthesis approaches. Type and mole ratio of monomers [15] [17], type of initiator [18], type of additive [10] [19] and type and mole percentage of the crosslinking agents [14] are the key factors in a synthesis reaction. Concentration, $\mathrm{pH}$, and type of salt solution are the most important properties of salt solutions. The most prominent features of medium that affect the hydrogel swelling behavior are pressure and temperature [10] [14].

Despite all experimental and field investigations carried out on the use of PPG samples to control unwanted water production [10] [11] and on poly(acrylamideco-acrylic acid) as a good commercial superabsorbent, no one examined the polymerization approach to optimize the swelling percentage of poly(acrylamideco-acrylic acid) in high salinity water $\left(\mathrm{CaCl}_{2}\right.$ salt water, $\left.200,000 \mathrm{ppm}\right)$. On the best of our knowledge, there is not any report in the literature that presents an efficient empirical correlation for water conformance based optimized key parameters affecting swelling capacity of poly(acrylamide-co-acrylic acid) in $\mathrm{CaCl}_{2}$ salt water as one of the most effective salts in the formation water. The aim of this work is to develop an optimum empirical correlation to predict the swelling capacity of PPGs, composed of AAm and AA, in $\mathrm{CaCl}_{2}$ salt water (200,000 ppm). To do so, the overall acceptable ranges of AAm/AA mole ratio [19], MBA mole 
percentage [19], and swelling time were examined as vital factors affecting the swelling percentage of PPGs in the salt water. Existing such empirical correlation that can optimize the swelling capacity of PPGs in the high salinity water can help producers to know how much PPGs have to be injected into the reservoir for water conformance.

Design of experiments (DOE) software is a benefit tool to elucidate and optimize the vital parameters affecting the desired experiments with minimum possible tests. There are many design methods in DOE. Response surface methodology (RSM), introduced by Box and Wilson [20], is one of the design approaches that examines key variables to optimize the desired responses. RSM has several mathematical and statistical techniques for optimization such as central composite design (CCD). Until now, several researchers used CCD approach to evaluate dominant parameters in synthesis experiments [21] [22] [23], to optimize chemical and physical properties of synthesized polymers [24] [25] and finally to propose a predictive mathematical model based on the performed synthesis experiments [26] [27] [28] [29]. To our purpose, we used CCD approach, Design- ${ }^{\circledR}$ V7 software, to design synthesis experiments. Mole ratio of AAm/AA, swelling time, and mole percentage of MBA were evaluated as the vital factors affecting the swelling percentage of PPGs.

In this study, we first synthesized 17 PPG samples by free radical approach based on CCD suggested runs. Then, we swelled PPGs in $\mathrm{CaCl}_{2}$ salt water and examined their swelling behavior. After that, we presented an empirical correlation that it can predict the swelling percentage of PPG samples. In the end, we evaluated the accuracy of our proposed correlation.

\section{Experiments}

\subsection{Materials}

Acrylamide (AAm, Sigma-Aldrich, >99\%) and acrylic acid (AA, Sigma-Aldrich, > 99\%) were two monomers used in this study. N,N'-methylenebisacrylamide (MBA, Sigma-Aldrich, $>99 \%)$ and sodium persulfate $\left(\mathrm{Na}_{2} \mathrm{~S}_{2} \mathrm{O}_{8}\right.$, Sigma-Aldrich, >98\%) were used as a crosslinking agent and an initiator, respectively. Distillated water was used as solvent in the synthesis experiments. $\mathrm{CaCl}_{2}\left(\mathrm{CaCl}_{2}\right.$, Merck Company, $99 \%$ ) salt solution with the concentration of 200,000 ppm was used in swelling experiments.

\subsection{Central Composite Design (CCD)}

To synthesis PPG samples and study their swelling behaviors, we considered the mole ratio of $\mathrm{AAm} / \mathrm{AA}$, mole percentage of $\mathrm{MBA}$ and swelling time as the dominant factors. Table 1 shows the mixing ranges of these key factors. As reported in [20], the mole ratio of AAm/AA and the mole percentage of the crosslinker agent in the copolymerization reaction are better to be in the range of 2 10 mole ratio and 4 - 20 mole percentage, respectively. In addition, several authors were used these mentioned ratios for AAm/AA and MBA [16] [30]. 
Table 1. The coded level of affected variables.

\begin{tabular}{ccccc}
\hline Process Variables & Designation & Units & -1 Level & +1 Level \\
\hline Acrylamide/Acrylic Acid & AAm/AA & $\ldots$ & 2.40 & 9.65 \\
N,N' methylenebisacrylamide & MBA & Mole $\%$ & 4 & 20 \\
Time & Time & min & 3.00 & 180.00 \\
\hline
\end{tabular}

Therefore, in this work the values of 2.41, 4.82, and 9.65 for AAm/AA mole ratio and the values of 4,16, 18 and 20 for MBA mole percentage were considered to cover the entire acceptable range of key parameters.

To achieve our aim, synthesizing an efficient set of PPG samples with an optimum swelling percentage, CCD suggested 17 experiments that three of them were replicated as the central points. Table 2 shows these proposed experiments.

\subsection{Synthesis Experiments}

To perform the 17 designed experiments, we first prepared crosslinking solutions. To do so, a certain amount of MBA, based on the suggested mole percentage by CCD, was fully dissolved in $1.0 \mathrm{~mL}$ of distillated water in a waterless volumetric flask $5.0 \mathrm{~mL}$ (borosilicate glass 3.3). Then, initiator solution was provided by carefully adding 0.1 grams of $\mathrm{K}_{2} \mathrm{~S}_{2} \mathrm{O}_{8}$ to $1.0 \mathrm{~mL}$ of distillated water in a dry volumetric flask $(5.0 \mathrm{~mL}$, borosilicate glass 3.3$)$. After that, 0.5 grams of AAm was quite solved in $1.0 \mathrm{~mL}$ of distillated water in a $10.0 \mathrm{~mL}$ falcontube for each experiment. $0.2 \mathrm{~mL}$ of the crosslinking solution was then meticulously added to the falcontube by using a microliter pipette $(100-1000 \mu \mathrm{L}$, WATSON NEXTY Japan). In the end, $0.1 \mathrm{~mL}$ of the initiator solution (by using the microliter pipette) was quickly augmented to the falcontube to begin the synthesis reaction. Immediately after that, the falcontube was completely closed and quickly put in the oil bath that it was heated to exactly $80^{\circ} \mathrm{C}$. The falcontubes were kept in the oil bath just for three hours.

\subsection{Swelling Experiments}

To investigate the swelling percentage of PPG samples, we first prepared $\mathrm{CaCl}_{2}$ salt solution with the concentration of exactly $200,000 \mathrm{ppm}$. Then for each synthesized PPG sample, we carefully weighed 2.0 grams of PPG and swelled it in 20 $\mathrm{mL}$ of the prepared $\mathrm{CaCl}_{2}$ salt solution. In a certain swelling time, which specified by CCD, swollen PPG sample was removed from the salt solution, its extra water was quickly taken with the use of a Whatman filter paper $(40: 8 \mu \mathrm{m})$, and carefully weighed by a digital balance (BP $210 \mathrm{~S}$, German). All the tests were carried out at room temperature. The swelling percentage of PPG samples was then calculated by Equation (1).

$$
\text { Swelling percentage }=\frac{m_{t}-m_{o}}{m_{o}} \times 100
$$

where, $m_{t}$ and $m_{o}$ are the wet and dry weight of PPG samples, respectively. 
Table 2. Central composite design.

\begin{tabular}{cccc}
\hline Run & AAm/AA (mole ratio) & MBA (mole \%) & Time (min) \\
\hline 1 & 4.82 & 14 & 90 \\
2 & 9.65 & 20 & 3 \\
3 & 4.82 & 20 & 90 \\
4 & 9.65 & 4 & 180 \\
5 & 4.82 & 4 & 90 \\
6 & 2.41 & 20 & 3 \\
7 & 9.65 & 4 & 3 \\
8 & 4.82 & 14 & 180 \\
9 & 2.41 & 4 & 180 \\
10 & 4.82 & 14 & 3 \\
11 & 2.41 & 14 & 90 \\
12 & 2.41 & 4 & 3 \\
13 & 4.82 & 14 & 90 \\
14 & 9.65 & 20 & 180 \\
15 & 4.82 & 14 & 90 \\
16 & 2.41 & 20 & 90 \\
17 & 9.65 & 16 & \\
\hline & & & 90 \\
\hline
\end{tabular}

\section{Results and Discussion}

In this study, we synthesized 17 PPG samples as previously described to examine the swelling behavior of hydrogels. To check the desirability of the synthesized PPG structure and its morphology, Fourier transform infrared spectroscopy (FT-IR, Shimadzu FTIR-8300) and scanning electron microscopy (SEM, VEGA3 TESCAN, at $20 \mathrm{kV}$ ) were fulfilled. As an example, FT-IR of PPG sample 5 was shown in Figure 1. According to Figure 1, O-H and $\mathrm{N}-\mathrm{H}$ stretching appeared in the range of $3100-3500 \mathrm{~cm}^{-1}$ confirms the formation of poly(AAm-co-AA). The asymmetric and symmetric $\mathrm{NH}_{2}$ stretching vibrations of acrylamide may be verified by two peaks appeared around 3395 and $3165 \mathrm{~cm}^{-1}$. The wide absorption bands from $3500 \mathrm{~cm}^{-1}$ to $2975 \mathrm{~cm}^{-1}$ can be attributed to the $-\mathrm{OH}$ bands of carboxylic groups. $\mathrm{CH}_{2}$ and $\mathrm{CH}$ stretching vibrations were revealed by happened picks at 2920 and $1850 \mathrm{~cm}^{-1}$, respectively. C-O stretching vibrations of carbonyl groups of acrylic acid and acrylamide became evident at 1645 and $1705 \mathrm{~cm}^{-1}$. The emerged band at $1120 \mathrm{~cm}^{-1}$ and the observed peak at $1440 \mathrm{~cm}^{-1}$, respectively, approved C-C stretching and C-N stretching vibrations.

Figure 2 exemplifies SEM image of PPG sample 5. There are several holes (1 $9 \mu \mathrm{m})$ in the structure of the examined PPG sample, which confirm the formation of a water-absorbing network in the PPG structure.

After reviewing the correctness of the performed synthesis experiments, we investigated the swelling behavior of PPG samples in $\mathrm{CaCl}_{2}$ salt solution. To do 


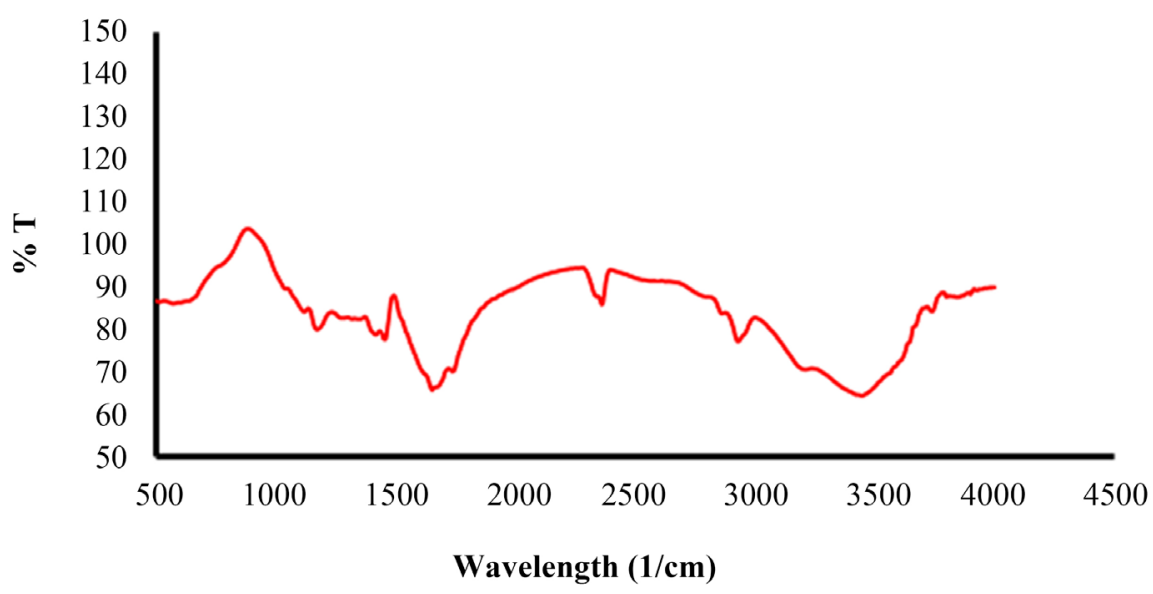

Figure 1. Fourier transform infrared spectroscopy of sample 1.

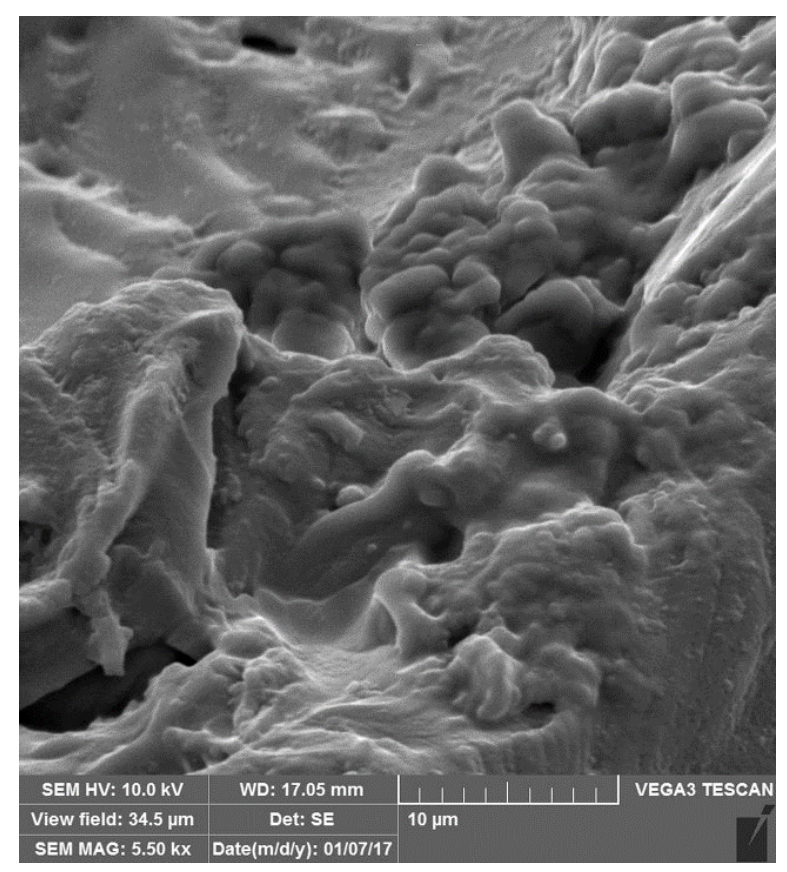

Figure 2. Scanning electron microscopy (SEM) image of sample 1.

so, the swelling experiments were carried out as previously described; the results were given to the software. CCD used these data to offer a mathematical model, which can predict the swelling percentage of PPG samples. Table 3 presents the results of swelling experiments. According to these results, the swelling percentage of PPG samples changes by all the mentioned key factors. Mole ratio of monomers, mole percentage of the crosslinking agent and swelling time are the influencing factors in PPG swelling behavior which are generally in line with the performed synthesis experiments reported by a variety of authors [14] [17] [30]. However, the trend of swelling changes of hydrogels against these dominant factors is not obvious in detail.

Based on the fit summary analysis, CCD suggested a quadratic model for the response. Table 4 shows the details of the analysis of variance (ANOVA) tab. 
Table 3. Central composite design matrix.

\begin{tabular}{|c|c|c|c|c|}
\hline Run & AAm/AA (mole ratio) & MBA (mole \%) & Time (min) & Swelling (\%) \\
\hline 1 & 4.82 & 14 & 90 & 240.73 \\
\hline 2 & 9.65 & 20 & 3 & 49.39 \\
\hline 3 & 4.82 & 20 & 90 & 228.91 \\
\hline 4 & 9.65 & 4 & 180 & 420.77 \\
\hline 5 & 4.82 & 4 & 90 & 267.21 \\
\hline 6 & 2.41 & 20 & 3 & 27.45 \\
\hline 7 & 9.65 & 4 & 3 & 65.78 \\
\hline 8 & 4.82 & 14 & 180 & 374.68 \\
\hline 9 & 2.41 & 4 & 180 & 157.54 \\
\hline 10 & 4.82 & 14 & 3 & 67.18 \\
\hline 11 & 2.41 & 14 & 90 & 69.10 \\
\hline 12 & 2.41 & 4 & 3 & 31.88 \\
\hline 13 & 4.82 & 14 & 90 & 248.88 \\
\hline 14 & 9.65 & 20 & 180 & 252.09 \\
\hline 15 & 4.82 & 14 & 90 & 258.05 \\
\hline 16 & 2.41 & 20 & 180 & 95.91 \\
\hline 17 & 9.65 & 16 & 90 & 199.68 \\
\hline
\end{tabular}

Table 4. ANOVA analysis (Partial Sum of Squares) for response surface quadratic model. (Response: swelling \%)

\begin{tabular}{cccccc}
\hline Source & Sum of Squares & df & Mean Square & F-Value & p-value \\
\hline Model & 11.8076 & 5 & 2.36152 & 154.4337 & $<0.0001$ \\
$A$-AAm/AA & 1.919111 & 1 & 1.919111 & 125.502 & $<0.0001$ \\
$B$-MBA & 0.262023 & 1 & 0.262023 & 17.1352 & 0.0016 \\
$\boldsymbol{C}$-Time & 6.48566 & 1 & 6.48566 & 424.1356 & $<0.0001$ \\
$A^{2}$ & 1.869314 & 1 & 1.869314 & 122.2455 & $<0.0001$ \\
$C^{2}$ & 0.448605 & 1 & 0.448605 & 29.33693 & 0.0002 \\
Residual & 0.168206 & 11 & 0.015291 & & \\
\hline
\end{tabular}

Since the vital properties of the model are in the statistically confident level of $95 \%$, the proposed model is significant; these properties are the P-value less than 0.0001 and the F-value equal to 154.43. On the basis of ANOVA results, $A, B, C$, $A^{2}$ and $C^{2}$ terms are dominant factors, which have P-value less than 0.0500 .

To adjust the response surface and the process parameters, CCD suggested a natural $\log$ transformation model. The value of R square was almost one (Table 5), which confirms the compatibility between the experimental results and the predicted values. Additionally, the slight difference between $\mathrm{R}^{2}$ and adjusted $\mathrm{R}^{2}$ (less than 0.2 ) verifies the accuracy of the proposed model. Equation (2) reveals the final coded mathematical model proposed by CCD in terms of coded factors. 
Table 5. Different statistical values from ANOVA analysis.

\begin{tabular}{cccc}
\hline Std. Dev. & 0.123659 & R-Squared & 0.985954 \\
\hline Mean & 4.89977 & Adj R-Squared & 0.97957 \\
C.V. \% & 2.523766 & Pred R-Squared & 0.96118 \\
PRESS & 0.464902 & Adeq Precision & 38.38345 \\
\hline
\end{tabular}

$$
\ln (\text { Swelling } \%)=+5.78+0.44 \times A-0.16 \times B+0.81 \times C-0.9 \times A^{2}-0.39 \times C^{2}
$$

where $A, B$, and $C$ are the previously defined parameters as key factors.

The diagnostic tab showed the validation of the model by the normal (Figure 3 ) and residual plot (Figure 4). Since the majority of the residuals were on the straight line, the error distribution of the performed experiments was normal. In addition, no unnatural schema or structure unfolded in the residual plot.

Which process parameter can more affect response surface? To find the answer, CCD changed one factor over its entire range while other factors were kept constant. The perturbation plot is a useful approach to compare the effect of vital factors on the response surface. It checks the deviation of each parameter from a reference point defined by CCD. The lower parameter deviation, the more effective parameter. Figure 5 shows that AAm/AA mole ratio and MBA mole percentage are more effective parameters than swelling time on the swelling percentage.

Figure 6 shows the difference between the actual and predicted values of the swelling percentage of PPG samples. Because a $45^{\circ}$-line traversed almost all points, there was a good agreement between actual and predicted values. This accordance reflects the accuracy of the proposed mathematical model.

Figure 7 reveals a three-dimensional plot of the swelling percentage against the AAm/AA mole ratio and MBA mole percentage. It is obvious that the swelling percentage curve has a maximum point against AAm/AA mole ratio and has downward trend against MBA mole percentage. Because of decreasing carboxylate groups existing in the AA structure, repulsive forces among them decrease and the swelling percentage increases. According to Lee and Yuan [29], there are two reasons for decreasing the swelling capacity by increasing AAm/AA mole ratio: first, increasing the mole ratio of AAm/AA increases the viscosity of the solution, limits the reactant movement and deactivates the growth of chains as soon as they form. Second, the reduction of carboxylate groups in copolymer chains decreases the osmotic pressure of the solution (p.9). For MBA concentration, on the other hand, the density of the brine solution increases by increasing the mole percentage of MBA that it reduces interpolation spaces among copolymer chains. This reduction forms a rigid structure, which cannot absorb large amounts of water and properly swell. The reduction of swelling capacity with a slight change in the mole percentage of the crosslinking agent is consistent with the previous findings [30].

The contour plot shown in Figure 8 confirms that PPG samples have a 


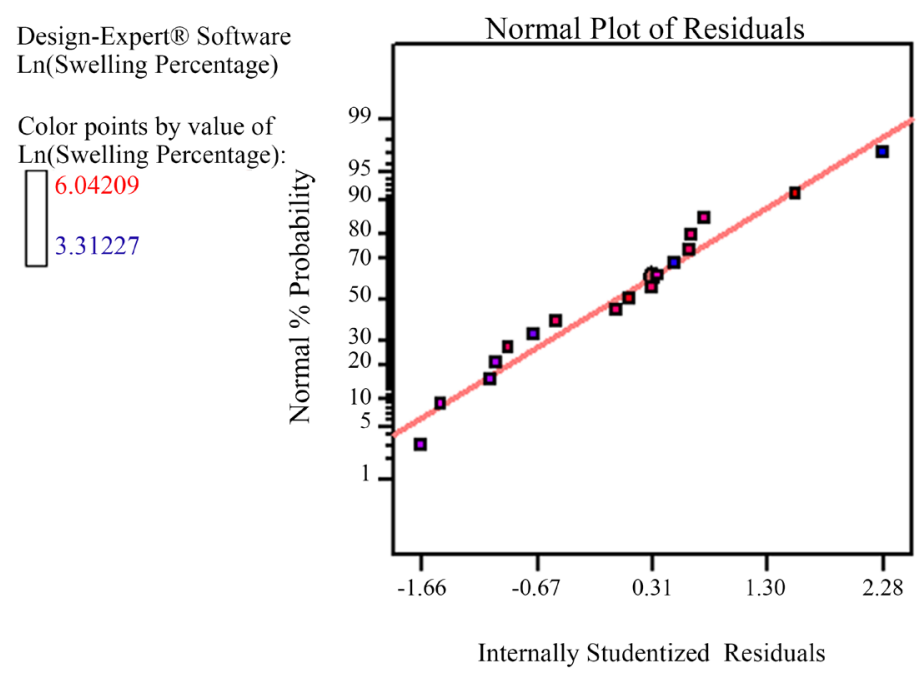

Figure 3. Normal probability plot of residuals.

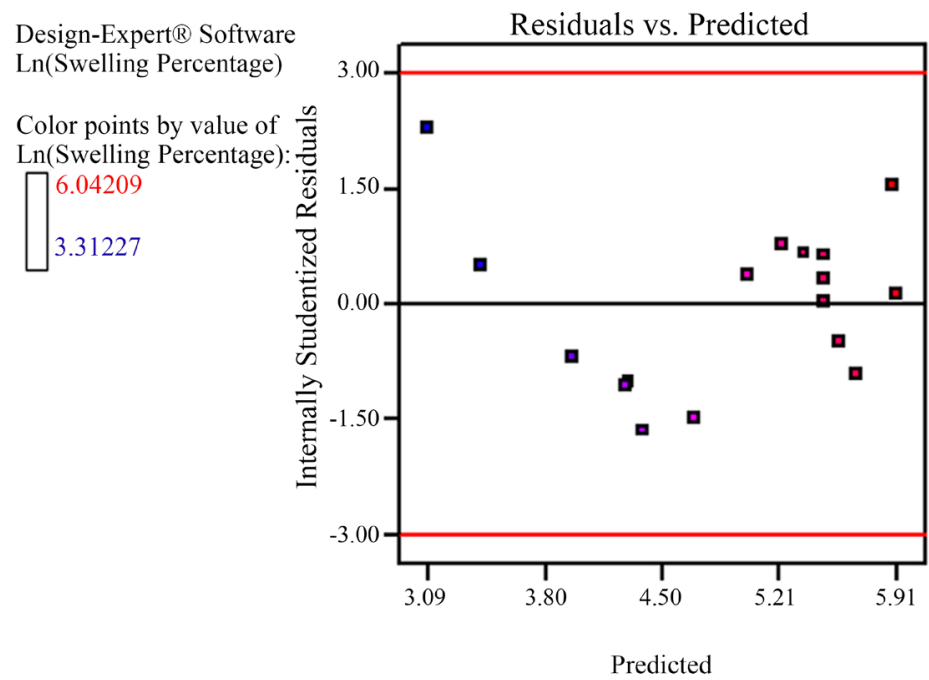

Figure 4. Residuals versus predicted values.

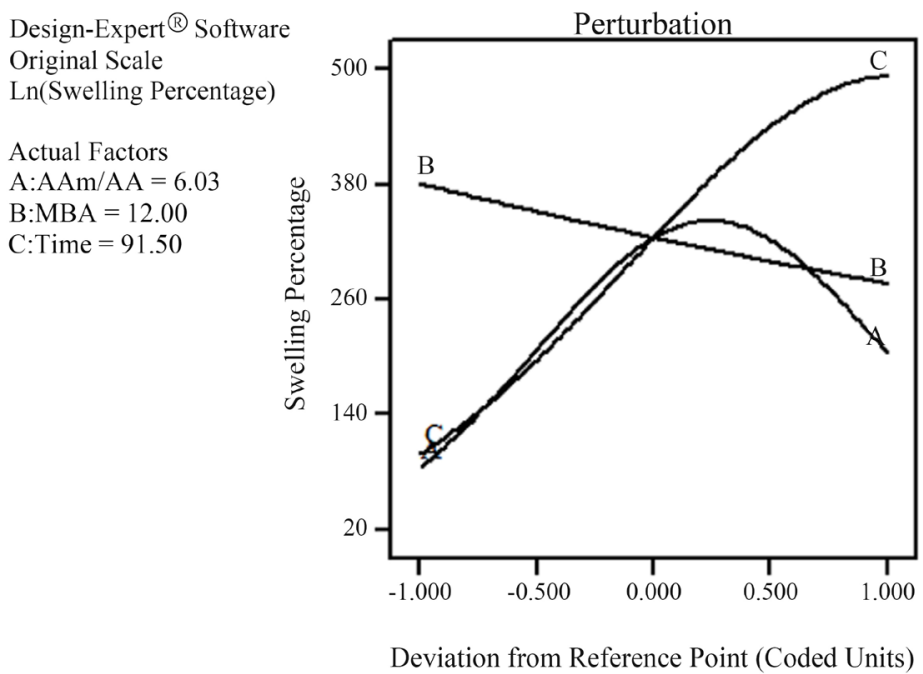

Figure 5. Perturbation plot. 


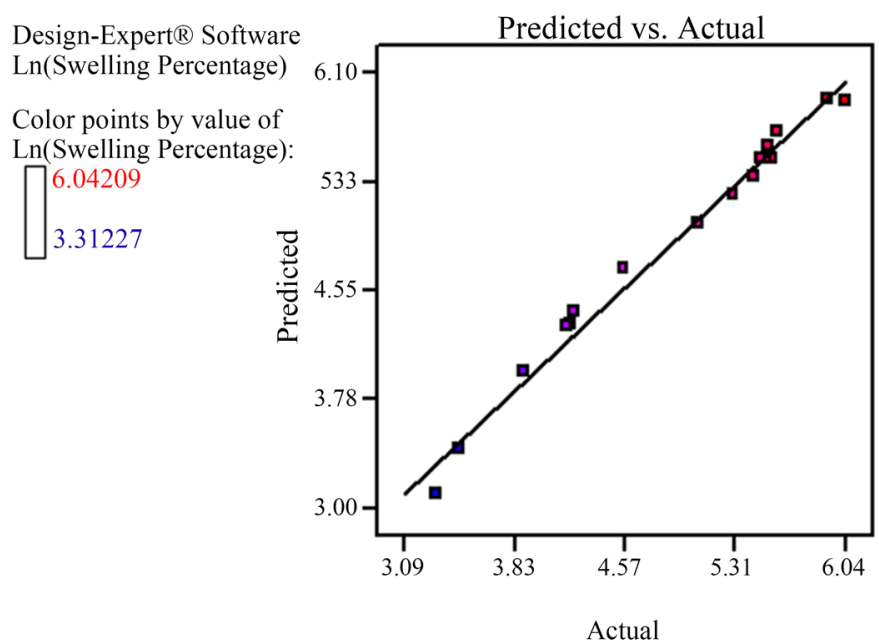

Figure 6. Predicted against actual responses.

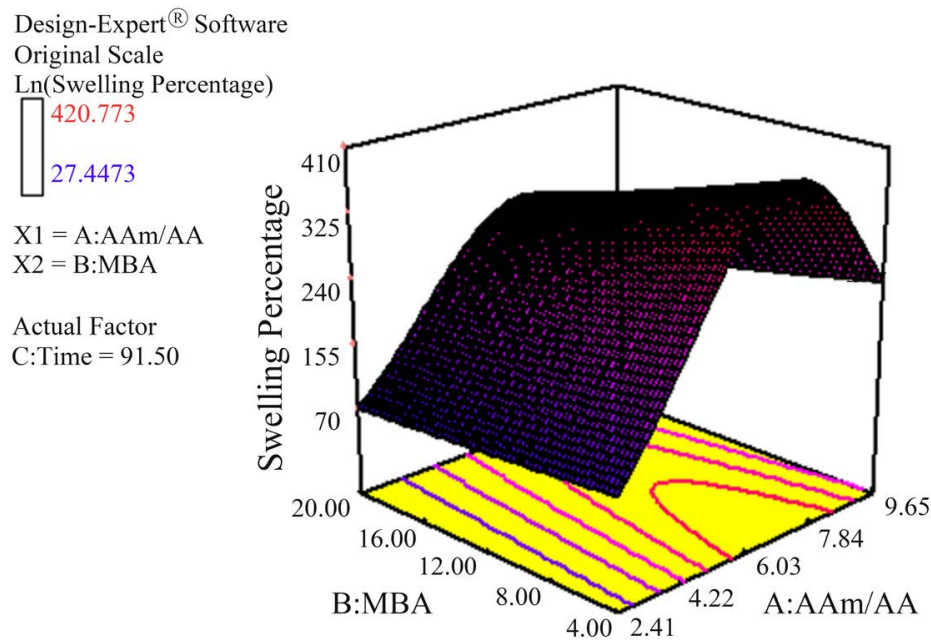

Figure 7. 3D surface graph of swelling percentage against the mole ratio of AAm/AA and mole percentage of MBA.

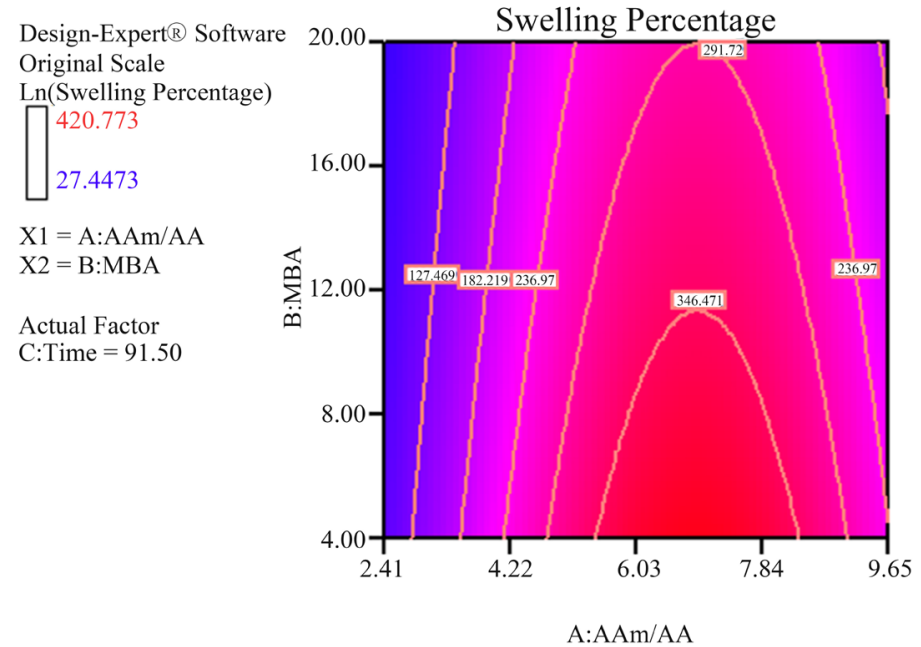

Figure 8. Contour plot of swelling percentage against the mole ratio of AAm/AA and mole percentage of MBA. 
maximum swelling percentage at the low mole percentage of MBA and the average mole ratio of AAm/AA.

To optimize the swelling percentage of PPG samples in $\mathrm{CaCl}_{2}$ salt water, in the numerical optimization tab, we considered the variation range of each key parameter in the same as its previously defined range (Table 1 ). In addition, the maximum value of the Ln (swelling percentage) was defined as the goal of optimization. CCD suggested three solutions to achieve this aim. Table 6 displays these proposed solutions.

To check the accuracy of the proposed optimization solutions, we performed the synthesis experiments based on the suggested values by CCD (Table 6). Table 7 illustrates that there was a good agreement between the predicted and obtained results. It means that the proposed solutions are acceptable.

Generally, we used CCD method to carry out synthesis experiments. The accuracy of the network structure of synthesized hydrogels was confirmed by FT-IR and SEM results. Swelling experiments revealed that the average amount of AAm/AA mole ratio, low amount of MBA mole percentage and high amount of swelling time could create optimal conditions to have a maximum swelling capacity. In the end, CCD proposed an empirical correlation, which could well predict the swelling behavior of PPG samples. Confirmatory experiments indicated that there were good agreements between actual and predicted results. Although there were some errors in suggested solutions for optimizing swelling conditions, they were negligible.

Table 6. Optimal conditions suggested by CCD.

\begin{tabular}{ccccc}
\hline Solutions & AAm/AA (mole ratio) & MBA (mole \%) & Time (min) & Desirability \\
\hline 1 & 6.94 & 4 & 180 & 1 \\
2 & 6.94 & 4.5 & 180 & 1 \\
3 & 6.94 & 5 & 180 & 1 \\
4 & 6.5 & 4 & 180 & 1 \\
5 & 7.3 & 4 & 180 & 1 \\
6 & 7 & 4 & 180 & 1 \\
\hline
\end{tabular}

Table 7. Obtained swelling percentage.

\begin{tabular}{cccccc}
\hline Run & $\begin{array}{c}\text { AAm/AA } \\
\text { (mole ratio) }\end{array}$ & MBA (mole \%) & Time (min) & $\begin{array}{c}\text { Predicted } \\
\text { swelling (S \%) }\end{array}$ & $\begin{array}{c}\text { Obtained } \\
\text { swelling (S \%) }\end{array}$ \\
\hline $\mathbf{1}$ & 6.94 & 4 & 180 & 609.98 & 607.54 \\
$\mathbf{2}$ & 6.94 & 4.5 & 180 & 603.92 & 600.76 \\
$\mathbf{3}$ & 6.94 & 5 & 180 & 597.92 & 598.32 \\
$\mathbf{4}$ & 6.5 & 4 & 180 & 603.10 & 601.65 \\
$\mathbf{5}$ & 7.3 & 4 & 180 & 603.61 & 601.98 \\
$\mathbf{6}$ & 7 & 4 & 180 & 609.67 & 606.96 \\
\hline
\end{tabular}




\section{Conclusion}

The present study awards a significant empirical correlation to predict swelling behaviors of PPGs in $\mathrm{CaCl}_{2}$ salt solution. The accuracy of the presented correlation was verified by several synthesis experiments. Although the synthesis mechanism of hydrogels remains to be determined, the suggested correlation can be used as a diagnostic test for many of polymer researchers. Since PPG treatment is one of the best cost-effective approaches for oil and gas producers to control unwanted water production, our study provides the framework for future studies to assess PPG performance in $\mathrm{CaCl}_{2}$ salt solution.

\section{Acknowledgements}

The authors are grateful to the Shiraz University and Enhanced Gas Condensate Recovery Research Group for supporting this research.

\section{Conflicts of Interest}

The authors declare no conflicts of interest regarding the publication of this paper.

\section{References}

[1] Portwood, J. (2005) The Kansas Arbuckle Formation: Performance Evaluation and Lessons Learned from More than 200 Polymer-Gel Water-Shutoff Treatments. SPE Production Operations Symposium, Oklahoma City, 16-19 April 2005. https://doi.org/10.2118/94096-MS

[2] Sydansk, R. and Southwell, G. (2000) More than 12 Years of Experience with a Successful Conformance-Control Polymer Gel Technology. SPE/AAPG Western Regional Meeting, Long Beach, 19-22 June 2000, 270-278. https://doi.org/10.2118/62561-MS

[3] Bai, B., Wei, M. and Liu, Y. (2013) Field and Lab Experience with a Successful Preformed Particle gel Conformance Control Technology. SPE Production and Operations Symposium, Oklahoma City, 23-26 March 2013. https://doi.org/10.2118/164511-MS

[4] Standnes, D.C. and Austad, T. (2000) Wettability Alteration in Chalk: 2. Mechanism for Wettability Alteration from Oil-Wet to Water-Wet Using Surfactants. Journal of Petroleum Science and Engineering, 3, 123-143. https://doi.org/10.1016/S0920-4105(00)00084-X

[5] Zhang, P. (2006) Water-Based EOR in Fractured Chalk; Wettability and Chemical Additives. Ph.D. Dissertation, Stavanger. ID: 935510.

[6] Stavland, A., Jonsbraten, H.C., Vikane, O., Skrettingland, K. and Fischer, H. (2011) In-Depth Water Diversion Using Sodium Silicate on Snorre-Factors Controlling In-Depth Placement. SPE European Formation Damage Conference, Noordwijk, 7-10 June 2011. https://doi.org/10.2118/143836-MS

[7] Willhite, G.P. and Pancake, R.E. (2008) Controlling Water Production Using Gelled Polymer Systems. SPE Reservoir Evaluation \& Engineering, 3, 454-465. https://doi.org/10.2118/89464-PA

[8] l-Muntasheri, G.A., Sierra, L., Garzon, F.O., Lynn, J.D. and Izquierdo, G.A. (2010) Water Shut-Off with Polymer Gels in a High Temperature Horizontal Gas Well: A 
Success Story. SPE Improved Oil Recovery Symposium, Tulsa, Oklahoma, 24-28 April 2010.

[9] Spildo, K., Skauge, A., Aarra, M.G. and Tweheyo, M.T. (2009) A New Polymer Application for North Sea Reservoirs. SPE Reservoir Evaluation \& Engineering, 3, 427-432. https://doi.org/10.2118/113460-PA

[10] Liu, Y., Zhu, M., Liu, X., Zhang, W., Sun, B., Chen, Y. and Adler, H.-J. P. (2006) High Clay Content Nanocomposite Hydrogels with Surprising Mechanical Strength and Interesting Deswelling Kinetics. Polymer, 1, 1-5. https://doi.org/10.1016/j.polymer.2005.11.030

[11] Bai, B., Huang, F., Liu, Y., Seright, R.S. and Wang, Y. (2008) Case Study on Prefromed Particle Gel for In-Depth Fluid Diversion. SPE Symposium on Improved Oil Recovery, Tulsa, Oklahoma, 20-23 April 2008. https://doi.org/10.2118/113997-MS

[12] Bai, B. and Zhang, H. (2011) Preformed-Particle-Gel Transport through Open Fractures and Its Effect on Water Flow. SPE Journal, 16, 388-400.

https://doi.org/10.2118/129908-PA

[13] Tongwa, P., Nygaard, R., Blue, A. and Bai, B. (2013) Evaluation of Potential Fracture-Sealing Materials for Remediating $\mathrm{CO}_{2}$ Leakage Pathways during $\mathrm{CO}_{2}$ Sequestration. International Journal of Greenhouse Gas Control, 18, 128-138. https://doi.org/10.1016/j.ijggc.2013.06.017

[14] Malana, M. A., Bukhari, J.-u.-D. and Zohra, R. (2014) Synthesis, Swelling Behavior, and Network Parameters of Novel Chemically Crosslinked Poly(acrylamideco-methacrylate-co-acrylic acid) Hydrogels. Designed Monomers and Polymers, 17, 266-274. https://doi.org/10.1080/15685551.2013.840501

[15] Othman, M.B.H., Akil, H.M., Osman, H., Khan, A. and Ahmad, Z. (2015) Synthesis, Characterisation and Thermal Properties of Hyperbranched Polyimide Derived from Melamine via Emulsion Polymerisation. Journal of Thermal Analysis and Calorimetry, 120, 1785-1798. https://doi.org/10.1007/s10973-015-4464-9

[16] Craciun, G., Manaila, E. and Stelescu, M.D. (2016) Electron Beam Synthesis and Characterization of Acrylamide/Acrylic Acid Hydrogels Using Trimethylolpropane Trimethacrylate as Cross-Linker. Journal of Chemistry, 2016, Article ID: 1470965.

[17] RPacheco, R.J., Silva, M., Sousa, R. and Freitas, R. (2014) Swelling Behavior of Polyacrylamide Hydrogels near Phase Transition. Materials Sciences and Applications, 5, 610-616. https://doi.org/10.4236/msa.2014.58063

[18] Kabiri, K., Omidian, H., Hashemi, S. and Zohuriaan-Mehr, M. (2003) Concise Synthesis of Fast-Swelling Superabsorbent Hydrogels: Effect of Initiator Concentration on Porosity and Absorption Rate. Journal of Polymer Materials, 1, 17-22.

[19] Tongwa, P. and Baojun, B. (2015) A More Superior Preformed Particle Gel with Potential Application for Conformance Control in Mature Oilfields. Journal of Petroleum Exploration and Production Technology, 2, 201-210.

https://doi.org/10.1007/s13202-014-0136-8

[20] Zheng, Y., Zhao, S., Zeng, A. and Guo, Y. (2012) The Application of Response Surface Methodology on the Synthesis of Grafted Polypropylene through the Solvothermal Route. Advances in Polymer Technology, 2, 109-117. https://doi.org/10.1002/adv.20242

[21] Zhao, Y., Fang, L. and Tan, T. (2006) Optimization of the Preparation of a Poly (Aspartic Acid) Superabsorbent Resin with Response Surface Methodology. Journal of Applied Polymer Science, 3, 2616-2622. https://doi.org/10.1002/app.23991 
[22] Hong, F.L., Peng, J. and Lui, W.B. (2011) Optimization of the Process Variables for the Synthesis of Starch-Based Biodegradable Resin Using Response Surface Methodology. Journal of Applied Polymer Science, 3, 1797-1804. https://doi.org/10.1002/app.32883

[23] Zare, Y., Garmabi, H. and Sharif, F. (2011) Optimization of Mechanical Properties of $\mathrm{PP} / \mathrm{Nanoclay} / \mathrm{CaCO}_{3}$ Ternary Nanocomposite Using Response Surface Methodology. Journal of Applied Polymer Science, 5, 3188-3200.

https://doi.org/10.1002/app.34378

[24] Cheng, W.Y., Peng, J. and Lui, W.B. (2011) Effects of $\alpha$-Amylase and Glycerol Levels on the Composition Optimization of Poly ( $\beta$-hydroxybutyrate co-valerate)/Starch Blended Biodegradable Resin Analyzed with Response Surface Methodology. Journal of Applied Polymer Science, 5, 2571-2578.

https://doi.org/10.1002/app.33326

[25] Biswas, S. and Mulaba-Bafubiandi, A.F. (2016) Optimization of Process Variables for the Biosynthesis of Silver Nanoparticles by Aspergillus wentii Using Statistical Experimental Design. Advances in Natural Sciences. Nanoscience and Nanotechnology, 4, 1-10.

[26] Bitaraf, M., Amoozadeh, A. and Otokesh, S. (2016) A Simple and Efficient One-Pot Synthesis of 1, 4-Dihydropyridines Using Nano-WO3-Supported Sulfonic Acid as an Heterogeneous Catalyst under Solvent-Free Conditions. Journal of the Chinese Chemical Society, 4, 336-344. https://doi.org/10.1002/jccs.201500453

[27] Ripoll, L. and Clement, Y. (2016) Polyamide Microparticles Containing Vitamin C by Interfacial Polymerization: An Approach by Design of Experimentation. Cosmetics, 4, 38. https://doi.org/10.3390/cosmetics3040038

[28] Raghupathy, R. and Amirthagadeswaran, K. (2014) Optimization of Casting Process Based on Box Behnken Design and Response Surface Methodology. International Journal for Quality Research, 4, 569-582.

[29] Işık, B. (2004) Swelling Behavior and Determination of Diffusion Characteristics of Acrylamide-Acrylic Acid Hydrogels. Journal of Applied Polymer Science, 2, 1289-1293. https://doi.org/10.1002/app.13270

[30] Lee, W.F. and Yuan, W.Y. (2000) Thermoreversible Hydrogels X: Synthesis and Swelling Behavior of the ( $\mathrm{N}$-isopropylacrylamide-co-sodium 2-acrylamido-2methylpropyl sulfonate) Copolymeric Hydrogels. Journal of Applied Polymer Science, 8, 1760-1768.

https://doi.org/10.1002/1097-4628(20000822)77:8<1760::AID-APP13>3.0.CO;2-J 